\title{
Article
}

\section{THE EFFECT OF TURBULENCE INTERMITTENCE ON THE EMISSION OF SOLAR ENERGETIC PARTICLES BY CORONAL AND INTERPLANETARY SHOCKS}

Kocharov, Leon, Laitinen, Timo Lauri Mikael and Vainio, Rami

Available at http://clok.uclan.ac.uk/10101/

Kocharov, Leon, Laitinen, Timo Lauri Mikael ORCID: 0000-0002-7719-7783 and Vainio, Rami (2013) THE EFFECT OF TURBULENCE INTERMITTENCE ON THE EMISSION OF SOLAR ENERGETIC PARTICLES BY CORONAL AND

INTERPLANETARY SHOCKS. Astrophysical Journal, 778 (1). L5. ISSN 2041-8205

It is advisable to refer to the publisher's version if you intend to cite from the work. http://dx.doi.org/10.1088/2041-8205/778/1/L5

For more information about UCLan's research in this area go to http://www.uclan.ac.uk/researchgroups/ and search for < name of research Group>.

For information about Research generally at UCLan please go to http://www.uclan.ac.uk/research/

All outputs in CLoK are protected by Intellectual Property Rights law, including Copyright law. Copyright, IPR and Moral Rights for the works on this site are retained by the individual authors and/or other copyright owners. Terms and conditions for use of this material are defined in the policies page. 


\title{
THE EFFECT OF TURBULENCE INTERMITTENCE ON THE EMISSION OF SOLAR ENERGETIC PARTICLES BY CORONAL AND INTERPLANETARY SHOCKS
}

\author{
Leon Kocharov ${ }^{1}$, Timo Laitinen ${ }^{2}$, and Rami Vainio ${ }^{3}$ \\ ${ }^{1}$ Sodankylä Geophysical Observatory (Oulu Unit), P.O. Box 3000, University of Oulu, FI-90014 Oulu, Finland \\ 2 Jeremiah Horrocks Institute, University of Central Lancashire, Preston PR1 2HE, UK \\ ${ }^{3}$ Department of Physics, P.O. Box 64, University of Helsinki, FI-00014 Helsinki, Finland \\ Received 2013 September 2; accepted 2013 October 14; published 2013 October 30
}

\begin{abstract}
Major solar energetic particle events are associated with shock waves in solar corona and solar wind. Fast scattering of charged particles by plasma turbulence near the shock wave increases the efficiency of the particle acceleration in the shock, but prevents particles from escaping ahead of the shock. However, the turbulence energy levels in neighboring magnetic tubes of solar wind may differ from each other by more than one order of magnitude. We present the first theoretical study of accelerated particle emission from an oblique shock wave propagating through an intermittent turbulence background that consists of both highly turbulent magnetic tubes, where particles are accelerated, and quiet tubes, via which the accelerated particles can escape to the non-shocked solar wind. The modeling results imply that the presence of the fast transport channels penetrating the shock and cross-field transport of accelerated particles to those channels may play a key role in high-energy particle emission from distant shocks and can explain the prompt onset of major solar energetic particle events observed near the Earth's orbit.
\end{abstract}

Key words: acceleration of particles - shock waves - Sun: coronal mass ejections (CME) - Sun: particle emission

Online-only material: color figures

\section{INTRODUCTION}

Diffusive shock acceleration operates in different astrophysical objects and can naturally generate a power-law energy spectrum of accelerated particles near and downstream of the shock wave (Krymsky 1977; Axford et al. 1977; Bell 1978; Blandford \& Ostriker 1978). Upstream of the shock front, the theoretical steady-state distribution of accelerated particles falls off exponentially with a scale length given by the ratio of the particle diffusion coefficients $D$ and the shock speed $U_{\mathrm{s}}$, both along the shock's normal (e.g., Toptygin 1985). The scale length $L_{D}=D / U_{\mathrm{s}}$ is expected to be energy dependent and should be small to make the shock acceleration fast. Hence, the standard model implies no particle escape to "upstream infinity." However, particle escape ahead of the shock is crucial for establishing a connection between the accelerated particles in astrophysical sources and the cosmic rays observed from Earth. For these reasons, the classic model of shock acceleration is often modified by introducing in an ad hoc manner a free-escape boundary at some location upstream (e.g., Reville et al. 2009; Caprioli et al. 2010).

Numerous solar observations link major solar energetic particle (SEP) events to shocks at/near the Sun (e.g., Lee 2006, and references therein). There have been many modeling efforts where the shock acceleration was heuristically included as a source term into the equation of SEP transport in the interplanetary medium, with the source spectrum assumed to be identical to the particle spectrum expected behind the shock (e.g., Heras et al. 1992; Kallenrode \& Wibberenz 1997). More advanced models adopt instead a free-escape boundary at a prescribed position upstream of the shock (e.g., Zank et al. 2000).

Until recently, in SEP studies the propagation across the mean magnetic field has often been neglected in favor of field-aligned propagation. The latter is considered substantially faster, with galactic cosmic ray observations (e.g., Burger et al. 2000), full-orbit simulations (e.g., Giacalone \& Jokipii
1999), and theoretical work (Matthaeus et al. 2003) showing the ratio of perpendicular and parallel diffusion coefficients as $\chi \equiv D_{\perp} / D_{\|} \sim 0.01$, depending on turbulence parameters. However, recent works have emphasized the significance of even weak cross-field propagation and field line meandering to the SEP event evolution in the interplanetary space (Zhang et al. 2009; Dröge et al. 2010; Laitinen et al. 2013).

In shock acceleration studies, the cross-field propagation of particles has been considered in the context of a particle crossing the oblique shocks propagating through a uniform background (Jones et al. 1993; Baring et al. 1995). In this Letter, we study the effect of perpendicular diffusion on particle acceleration and transport in the case of a spatially intermittent pre-shocked background.

\section{OBSERVATIONAL AND THEORETICAL MOTIVATION}

In major (gradual) SEP events, the emission of deka-MeV protons develops on the time scale of $\sim 10-60$ minutes and then continues for many hours (e.g., Solar and Heliospheric Observatory data presented by Kocharov et al. 2007a, 2012). The proton flux anisotropy data reveal the long-lasting streaming of deka-MeV protons far upstream of the coronal-mass-ejectiondriven shock that still expands near the Sun. The same anisotropy measurements, as well as the interplanetary magnetic field data, indicate that the rate of pitch-angle scattering of deka$\mathrm{MeV}$ protons is highly variable and may differ by an order of magnitude in two neighboring magnetic flux tubes of the solar wind (Torsti et al. 2004; Kocharov et al. 2007b), while an overall spread of the scattering frequency (the fitted mean free path) in different SEP events comprises at least two orders of magnitude.

Figure 1 illustrates a simple model employed to study how the strong intermittence of the particle mean free path could affect the particle acceleration in a shock wave and how the accelerated particle could escape to far upstream of the shock. Particles are accelerated mainly in Tube A, where scattering is frequent 


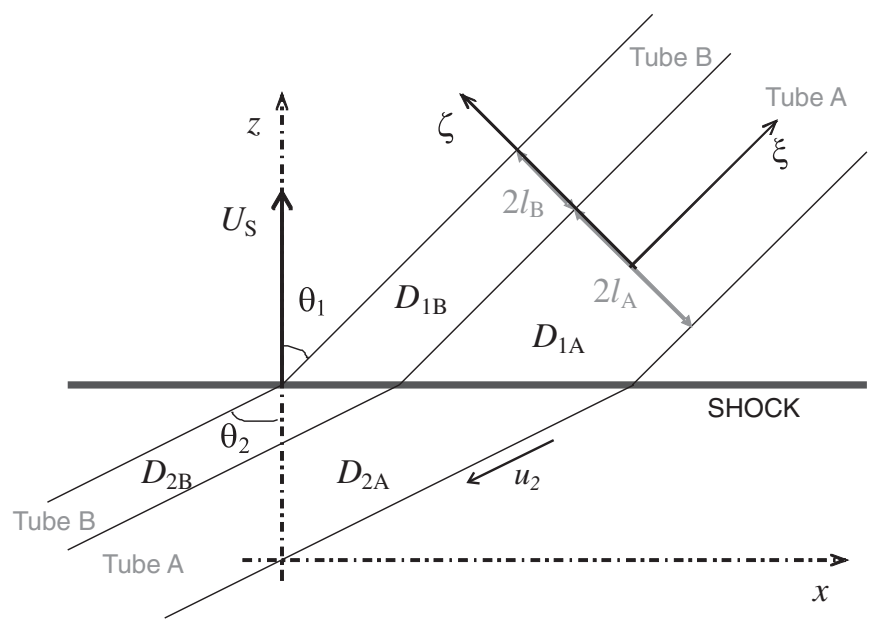

Figure 1. Particle acceleration and transport model. Particles are promptly accelerated by the shock in Tube A, where scattering is frequent, and can escape to far upstream of the shock in the neighboring tube of slow scattering, Tube B.

and hence the shock acceleration is fast. Then the accelerated particles, which are abundant in the vicinity of the shock front and behind it in Tube A, can diffuse across the magnetic field lines into the neighboring Tube $\mathrm{B}$, where the turbulence is much weaker than in Tube A. Particle transport in Tube B is fast and the accelerated particles can overtake the shock and escape to the far upstream solar wind. For such a scenario to be effective, we have to assume that advection with a plasma flow of speed $u_{2}$ behind the shock over the perpendicular transport time $\left[\tau_{\perp 2}=l_{2}^{2} /\left(4 D_{\perp 2}\right)\right]$ does not exceed the characteristic parallel diffusion length in Tube B $\left(L_{D}^{(B)}=D_{\| 2}^{(B)} / u_{2}\right)$, i.e., the shockaccelerated particles move from Tube A to Tube B sufficiently close to the shock to return to it in Tube B:

$$
\tau_{\perp 2} u_{2}<\frac{D_{\| 2}}{u_{2}} X
$$

where $l_{2}, D_{\perp 2}$, and $D_{\| 2}$ are, respectively, the half-width of Tube A and the perpendicular and parallel diffusion coefficients in the tube behind the shock (we omit the tube index " $\mathrm{A}$ " but keep the index " $\mathrm{B}$ " in all expressions), $X$ is the ratio of parallel diffusion coefficients between the tubes: $X \equiv$ $D_{\| i}^{(B)} / D_{\| i}^{(A)} \equiv D_{\| i}^{(B)} / D_{\| i} \gg 1, i=1,2$ for the shock upstream and downstream regions, respectively, and $u_{i}$ is the plasma flow speed in the de Hoffmann-Teller frame (de Hoffmann \& Teller 1950). The condition can be expressed in terms of the characteristic time of particle acceleration in the shock of a compression ratio $\sigma, \tau_{a} \approx 3 \sigma D_{\| 1} /\left[u_{1}^{2}(\sigma-1)\right]$ (Drury 1983):

$$
\frac{l_{2}}{U_{\mathrm{s}} \tau_{a}}<\frac{\sigma-1}{3 \sigma} \frac{D_{\| 2}}{D_{\| 1}} \frac{u_{1}}{u_{2}} \frac{2 \sqrt{\chi}}{\cos \theta_{1}} \sqrt{X} .
$$

where $\chi \equiv D_{\perp i} / D_{\| i}, U_{\mathrm{s}}$ is the shock speed and $\theta_{1}$ is the shock angle that is assumed to be not very large: $\tan \theta_{1} \ll \chi^{-1 / 2}$. Then we impose a second limitation:

$$
\tau_{\perp 2}>\tau_{a},
$$

which allows particles to be accelerated before they leave the acceleration Tube A. This limitation can be re-cast into a form parallel to Equation (2):

$$
\frac{l_{2}}{U_{\mathrm{s}} \tau_{a}}>\sqrt{\frac{\sigma-1}{3 \sigma} \frac{D_{\| 2}}{D_{\| 1}}} \frac{2 \sqrt{\chi}}{\cos \theta_{1}} .
$$

Finally, by combining both limitations together, one can estimate the transverse jump of diffusion coefficient required to get particles both accelerating at the shock and escaping to the upstream solar wind:

$$
X>\frac{3 \sigma}{\sigma-1} \frac{D_{\| 1}}{D_{\| 2}}\left(\frac{u_{2}}{u_{1}}\right)^{2} .
$$

For the parallel shock of compression ratio $\sigma=4$ and the diffusion coefficient jump at the shock front $D_{\| 1} / D_{\| 2} \approx 20$ (Vainio \& Schlickeiser 1999), Equation (5) yields the required transverse jump magnitude $X>5$. For an oblique shock with $\theta_{1}=45^{\circ}$ and $\sigma=3.5$, the transverse jump of the diffusion coefficient will be $X>50$. In view of in situ SEP and plasma data, these values do not look impossible.

\section{NUMERICAL MODEL}

We re-cast the classic diffusion-convection equation of cosmic ray transport (e.g., Toptygin 1985) in terms of the number of particles per unit of magnetic tube length, $N(\xi, i, E, t)=$ $F(\boldsymbol{r}(\xi, i), E, t) \Phi / B(\boldsymbol{r}(\xi, i), t)$, where $F(\boldsymbol{r}, E, t)$ is volumetric number density of particles of energy $E, \xi$ is the curvilinear coordinate along the magnetic line $i$, and $\Phi$ is the normalization magnetic flux. This leads to a field-alighted form of the standard transport equation (similar to Equation (10) by Kocharov et al. 2012, but with perpendicular diffusion term and no stochastic acceleration added). The equation is solved with numerical simulations of random walks and advection of "Monte Carlo particles" in the $\xi-\zeta-E$ space, where $\zeta$ is the local coordinate perpendicular to the magnetic field (Figure 1). Perpendicular diffusion proceeds in the coordinate $\zeta$ :

$$
\left(\frac{\partial N}{\partial t}\right)_{\perp}=\frac{1}{B} \frac{\partial}{\partial \zeta} D_{\perp} \frac{\partial}{\partial \zeta} N B
$$

but we treat the perpendicular diffusion not in terms of the coordinate $\zeta$, but in terms of the coordinate $\zeta_{o}$ that the current magnetic field line has at the outer (upstream) boundary of the simulation box. For the system shown in Figure 1, those coordinates relate to each other as $\zeta=\zeta_{o} B_{o} / B(\xi)$, where $B_{o}$ is the magnetic field intensity far upstream of the shock. Hence, the cross-field transport will be solved from the equation

$$
\left(\frac{\partial N}{\partial t}\right)_{\perp}=\frac{\partial}{\partial \zeta_{o}} D_{\perp}\left(\xi, \zeta_{o}\right)\left[\frac{B(\xi)}{B_{o}}\right]^{2} \frac{\partial N}{\partial \zeta_{o}}
$$

in a box on the $\xi-\zeta_{o}$ plane.

The shock is modeled as a narrow continuous compression of width $\Delta_{\mathrm{S}}$ in such a way that near the shock location the plasma flow speed in the de Hoffmann-Teller frame is of the form:

$$
u(\xi, t)=\frac{1}{2}\left\{u_{1}+u_{2}+\left(u_{1}-u_{2}\right) \tanh \left[\left(\xi-\xi_{s}\right) / \Delta_{\mathrm{s}}\right]\right\},
$$

where the indices " 1 " and " 2 " refer to upstream and downstream regions, respectively; $\xi_{\mathrm{s}}=\xi_{\mathrm{s}}(t)$ is the shock's coordinate along the magnetic field line. The shock's Alfvénic Mach number and plasma beta for the Rankine-Hugoniot jump conditions are, respectively, 5 and 0.3 . We assume that magnetic field intensity and diffusion coefficients as functions of $\xi$ change in the shock front in the same way as the hydrodynamic speed does, but with their own jump ratios. In the direction perpendicular to the magnetic field, we consider two adjacent (half) tubes, Tube A and Tube $\mathrm{B}$, of the upstream half-width $l_{1 A}+l_{1 B}=R_{\odot}$ and 


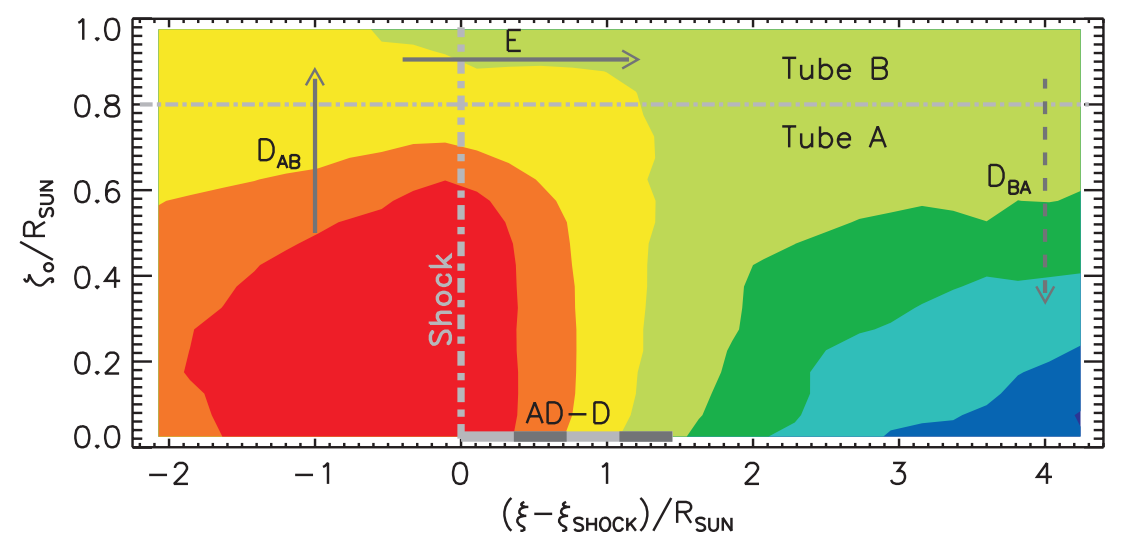

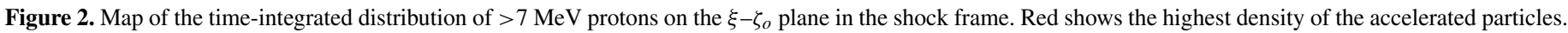

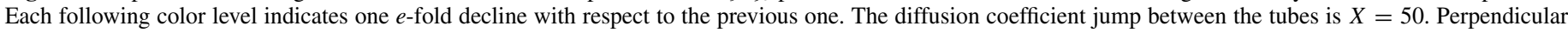
diffusion is energy dependent: $D_{\perp}(E)=0.04 D_{\|}(E)$. The integration time is 30 minutes.

$l_{1 A} / l_{1 B}=4$. The tubes differ in their intensity of contained Alfvén waves for the energetic particle scattering. The $\zeta_{0^{-}}$ dependence of the diffusion coefficient is again in the hyperbolic tangent form with the transition width $\Delta_{\mathrm{m}}=0.1 R_{\odot}$ at the boundary between the magnetic tubes A and B.

For the goals of this first study, we consider a planar shock of the speed $U_{\mathrm{s}}=2 \times 10^{3} \mathrm{~km} \mathrm{~s}^{-1}$ that starts at the "height" $z_{o}=0.1 R_{\odot}$ and travels away from the reference plane $(z=0)$ over the 30 minute time span in a uniform magnetic field that makes, with the shock velocity, the upstream angle $\theta_{1}=45^{\circ}$ (Figure 1). In the reference frame, plasma is at rest upstream of the shock, then the hydrodynamic speed jumps up at the shock front and returns back to zero at $z=0$. We assume a linear change in the hydrodynamic speed between $z=0$ and the shock front.

The particle simulation box extends along the magnetic field from the reference plane to an outer boundary placed at the "height" $z=14 R_{\odot}$ and in the perpendicular to magnetic field direction from $\zeta_{o}=0$ to $\zeta_{o}=R_{\odot}$. It includes only a half of each tube. In the case of parallel shocks, the spatial distribution of accelerated particles in either tube is symmetric with respect to the tube's axis, while some asymmetry of the transverse distribution may be expected for particles accelerated by oblique shocks. However, we neglect possible transverse-asymmetry effects on the particle escape along the magnetic field lines. In such symmetric approximation, particles are bounced back at both side boundaries, $\zeta_{o}=0$ and $\zeta_{o}=R_{\odot}$. The inner, "solar" boundary at $z=0$ is closed, while the outer boundary at $z=14 R_{\odot}$ is open into the "interplanetary" space.

At each Monte Carlo time step, $\delta t$, a particle experiences a random walk in the transverse coordinate: $\delta \zeta_{o}=\sqrt{2 D_{I} \delta t} R_{\mathrm{G}}+$ $\left(\partial D_{I} / \partial \zeta_{o}\right) \delta t$, with $D_{I}=\left(B / B_{o}\right)^{2} D_{\perp}$ and $R_{\mathrm{G}}$ being a normally distributed random number. Treatment of the particle transport in the parallel to magnetic field direction is similar to our previous modeling (Kocharov et al. 2012) and includes parallel diffusion and two advection processes, due to the plasma flow and the magnetic focusing, while the shock acceleration arises via the divergence of plasma flow, as does the downstream adiabatic deceleration. Compared to the previous modeling, we have to additionally include the $\xi$-coordinate change of a geometric nature, $\delta \xi_{\mathrm{g}}$, because the coordinate mesh is defined by now changeable magnetic field lines. The coordinate grid at the particle location may be shifted due to the elongation of a given magnetic field line caused by advancing of the shock or due to the particle moving in the course of perpendicular diffusion to another magnetic field line of a different length.

The parallel diffusion coefficient value in Tube A upstream of the shock is adopted at a sufficiently small level to get protons accelerated to $\approx 100 \mathrm{MeV}$ within the first 30 minutes: $D_{\| 1}(0.1 \mathrm{MeV})=2 \times 10^{17} \mathrm{~cm}^{2} \mathrm{~s}^{-1}$, with the energy dependence $D_{\|} \propto E^{0.75}$ corresponding to the turbulence spectrum index $S=1.5$ (e.g., Toptygin 1985). Parallel diffusion in Tube B is much faster: $D_{\| 1}^{(B)}=X D_{\| 1}$ with $X=50$ or 100 . Parallel diffusion coefficients behind the shock, $D_{\| 2}$ and $D_{\| 2}^{(B)}$, are smaller by a factor of 20 compared to their upstream values. Two models of perpendicular diffusion are considered. In the first model, the ratio of diffusion coefficients, $\chi \equiv D_{\perp}(E) / D_{\|}(E)$, is independent of energy. We adopt $\chi=0.04$. The second model suggests the perpendicular diffusion coefficient itself is independent of energy: $D_{\perp}=0.04 D_{\|}(50 \mathrm{MeV})$.

We assume the seed particle population for the shock acceleration is equally available at all locations, with an exponential energy distribution: $N_{\text {seed }} \propto \exp (-E / 0.3 \mathrm{MeV})$. Seed particles above the acceleration threshold undergo the acceleration. A particle splitting technique is applied to enhance the statistics at the high energy end. Particles are registered at each Monte Carlo time step to obtain their distribution in two spatial coordinates, energy and time. We separately register particles escaping at the top of the simulation box to estimate the energy spectrum of particle emission to the shock's "upstream infinity."

\section{MODELING RESULTS AND DISCUSSION}

Figure 2 exemplifies the time-integrated spatial distribution of accelerated protons in the system of the shock. Particles are accelerated near the shock in Tube A, where their acceleration is fast due to the small value of the parallel diffusion coefficient. The upstream distribution in the center of Tube A represents a balance of particle diffusion from the shock and their advection in the opposite direction. Here we obtain the exponential distribution identical to the distribution known for uniform upstream plasma (e.g., Toptygin 1985), as illustrated by the scale bar "AD-D." However, closer to the boundary with Tube B, some downstream particles can leave the Tube A via perpendicular diffusion (arrow " $\mathrm{D}_{\mathrm{AB}}$ "). This is what was assumed for the preliminary estimates in Section 2. In addition, we observe some diffusion from Tube A to Tube B in the nearest upstream region, at $\xi-\xi_{\mathrm{s}}<1.5 R_{\odot}$ and $\zeta_{o}=0.6-0.8$. Upon arrival at Tube $\mathrm{B}$, 

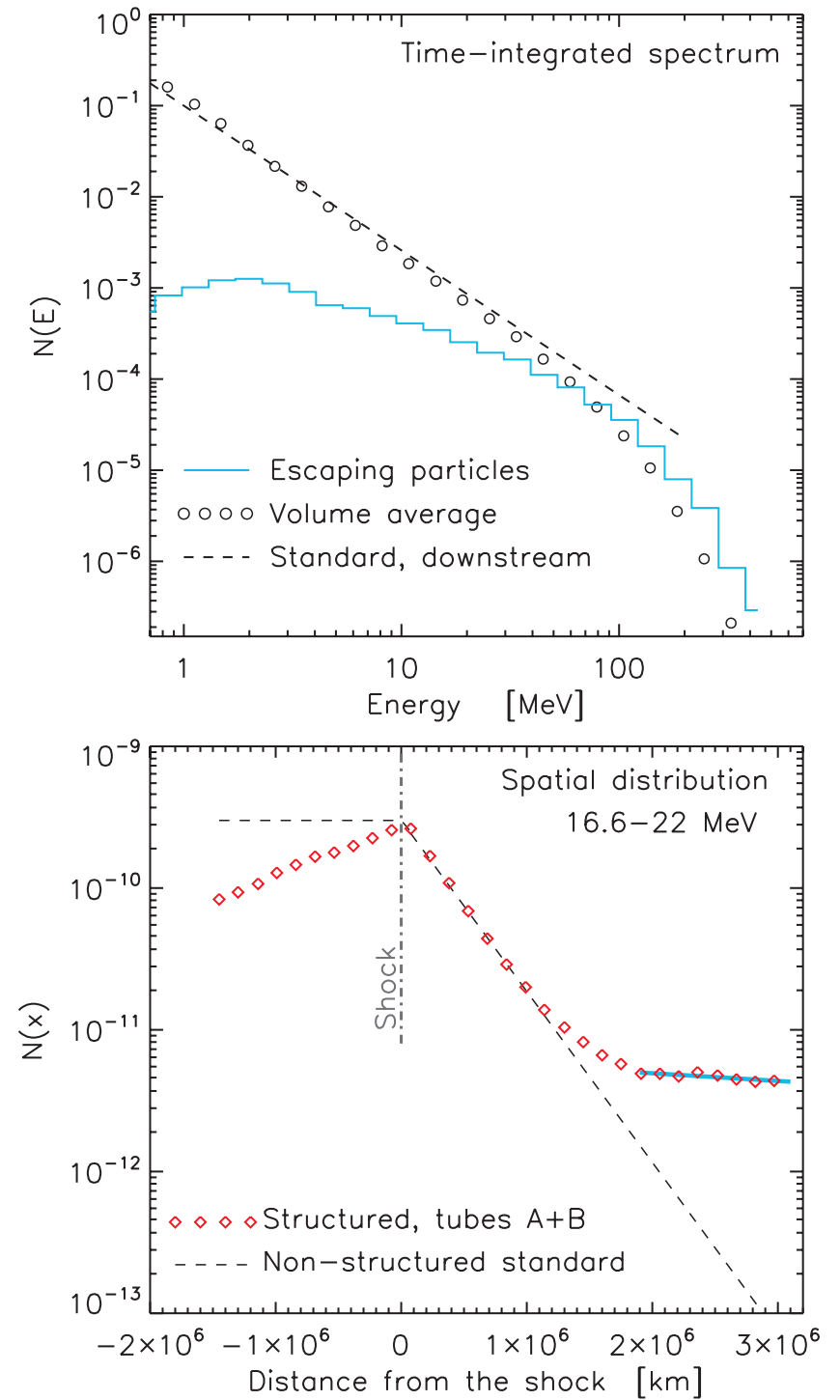

Figure 3. Energy spectrum of shock-accelerated protons in the case of energydependent perpendicular diffusion, $D_{\perp}(E) \propto D_{\|}(E)$. Upper panel: energy spectrum of accelerated protons inside the simulation box and spectrum of protons escaping through the open boundary far upstream of the shock (points and histogram, respectively). Shown with a dashed line is the typically expected, classic power-law spectrum that would eventually be formed in the shock downstream region in the case of a shock propagating through uniform (nonstructured) plasma: $N(E) \propto E^{-\gamma}, \gamma=(\sigma+0.5) /(\sigma-1)$ with $\sigma=3.5$. Lower panel: spatial distribution of $16.6-22 \mathrm{MeV}$ protons in the shock frame, summed over tubes A and B and time-integrated (points). The dashed line shows the exponential distribution expected upstream of the shock in the case of uniform plasma, which implies no particle escape to the shock's upstream infinity. The diffusion coefficient jump between the tubes is $X=100$. The integration time is 30 minutes.

(A color version of this figure is available in the online journal.)

the accelerated particles can easily escape to far upstream of the shock, as illustrated by arrow "E." Note that some particles may return to Tube A after the perpendicular diffusion upstream of the shock (dashed arrow " $\mathrm{D}_{\mathrm{BA}}$ ").

The upper panels of Figures 3 and 4 show average spectra of accelerated particles inside the system (points), which are contributed mainly by particles downstream of the shock. Those spectra are close to the standard spectrum that is the steadystate spectrum at the shock and downstream of it in the case of uniform plasma background. In contrast to the standard model,
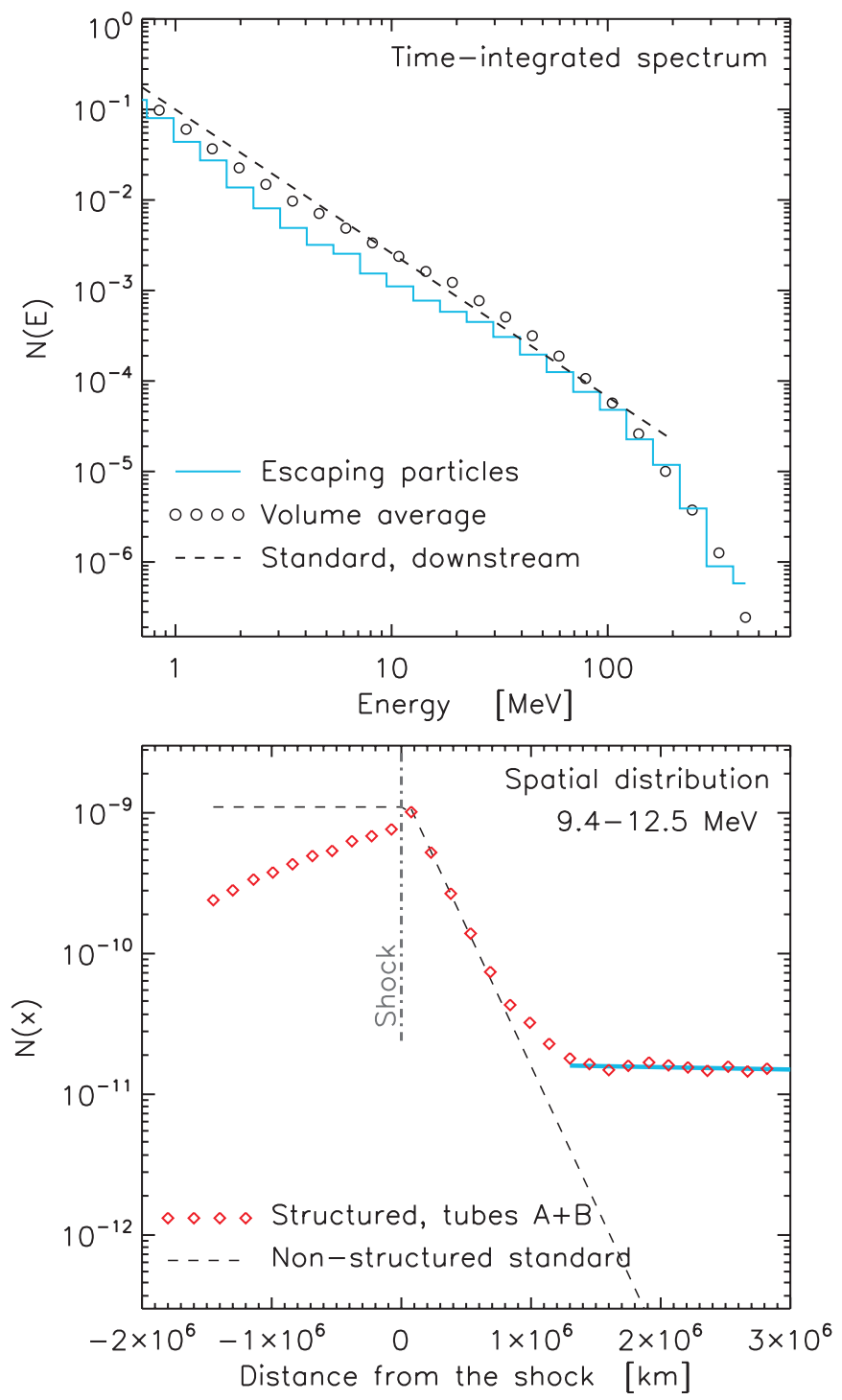

Figure 4. Energy spectrum of shock-accelerated protons in the case of energy-independent perpendicular diffusion. Upper panel: energy spectrum of accelerated protons inside the simulation box and spectrum of protons escaping far upstream of the shock (points and histogram, respectively). The classic downstream spectrum is shown by the dashed line. Lower panel: spatial distribution of 9.4-12.5 MeV protons in the shock frame, summed over tubes $\mathrm{A}$ and $\mathrm{B}$ and time-integrated (points). The dashed line shows the classic exponential distribution. The new model allows downstream particles to escape to the shock's far upstream region (blue line). The diffusion coefficient jump between the tubes is $X=100$. The integration time is 30 minutes.

(A color version of this figure is available in the online journal.)

the presence of the weakly turbulent Tube B in our model allows particles to escape upstream of the shock. The escaping particle spectrum (histogram) depends, however, on assumptions on the energy dependence of the perpendicular diffusion coefficient, $D_{\perp}$ (upper panel of Figure 3 versus corresponding panel of Figure 4).

The lower panels of Figures 3 and 4 show simulated spatial distributions of accelerated particles with respect to the shock front after integration over both time and transverse coordinate $\zeta_{o}$. In the standard case of uniform upstream plasma, the proton distribution would decline exponentially with distance from the shock upstream of it (shown by the dashed line in both figures). The characteristic length of the standard distribution is $L_{D}=u_{1} / D_{\| 1}(E)$. In our model of the intermittent upstream 
turbulence, the escaping particle tail is clearly seen in both figures (indicated with blue line). It looks much like the result of an escape boundary that would be placed at $\approx 2 R_{\odot}$, despite no escape boundary being assumed there (cf. Zank et al. 2000).

Accelerated protons streaming from the shock may themselves excite the resonant Alfvén waves (e.g., Lee 2005; Vainio \& Laitinen 2007), which have not been incorporated into the current model. A proton scattered by an Alfvén wave gains/ loses the amount of energy $d E_{\mathrm{p}}= \pm v_{\mathrm{A}} d p_{\|}$in the plasma frame, where $v_{A}$ is the Alfvén speed, $p_{\|}$is the particle momentum projection onto the large-scale magnetic field and the sign depends on the wave propagation direction along the magnetic field (e.g., Afanasiev \& Vainio 2013). The wave energy change is correspondingly $d E_{\mathrm{w}}=-d E_{\mathrm{p}}$. Energetic protons injected from the shock to its upstream region can amplify the forward propagating Alfvén waves and thus could enhance scattering frequency. The particle scattering, however, reduces the proton flux anisotropy and thus decreases the wave amplification rate. We estimate the rate of the wave energy change in unit volume as

$$
\frac{d w_{\mathrm{w}}}{d t} \approx \frac{D_{\|}}{v L_{n}} \frac{v_{\mathrm{A}} p}{\Lambda_{\|} / v} n_{\mathrm{p}},
$$

where $n_{\mathrm{p}}$ is the number density of the energetic protons; $D_{\|} /\left(v L_{n}\right)$ is an estimate of the proton flux anisotropy with $L_{n}$ being the characteristic length scale of the proton number density distribution; $D_{\|}=\Lambda_{\|} v / 3$. In context of our model, $L_{n}=L_{D}$. The proton amplified waves can be neglected when the characteristic time of the wave amplification, $\tau_{\mathrm{w}} \equiv$ $w_{\mathrm{w}} /\left(d w_{\mathrm{w}} / d t\right)$, is large compared to the shock transit time over the spatial scale of the energetic proton distribution: $\tau_{\mathrm{w}}>\tau_{\mathrm{s}} \equiv L_{D} / U_{\mathrm{s}}$, which implies that the energy density of energetic protons are sufficiently small:

$$
w_{\mathrm{p}}<M_{\mathrm{A}} w_{\mathrm{w}},
$$

where $w_{p}$ is the energy density of accelerated protons, $M_{\mathrm{A}} \equiv$ $U_{\mathrm{s}} / v_{\mathrm{A}}$ is the Alfvénic Mach number of the shock, and $w_{w}$ is the energy density of the resonant waves that can be expressed in terms of the model parameter $D_{\| 1}$ (Toptygin 1985; Schlickeiser \& Steinacker 1989):

$$
w_{\mathrm{w}}\left(k \geqslant R_{\mathrm{L}}^{-1}\right) \approx \frac{v R_{\mathrm{L}}}{2 D_{\| 1}} w_{B}
$$

where $R_{\mathrm{L}}$ is the Larmour radius of the energetic proton and $w_{B}$ is the energy density of the regular magnetic field, $w_{B}=B^{2} /(8 \pi)$.

As in situ observations indicate, the energetic proton density is highly variable, depending on the SEP event phase, the event overall magnitude and the proton energy range. At high energies $(\gtrsim 100 \mathrm{MeV}$ ), wave generation may be inefficient since the energy content in those protons is low. At lower energies, on the other hand, it takes time for the waves to grow, and the time-integrated growth rate during wave enhancement is given by the total time-integrated net flux of particles escaping to the ambient medium (see Vainio 2003). Therefore, we may obtain a prompt escaping population from the shock from the time when the waves have not yet grown to high amplitudes. After that, the particle escape via transport Tube B will continue with the intensity being fixed by the self-generated waves at a saturation level $\left(w_{\mathrm{p}} \approx M_{\mathrm{A}} w_{\mathrm{w}}\right)$. This would be similar to the streaminglimited intensities of SEPs observed in the interplanetary space (Reames \& Ng 1998).

\section{CONCLUSION}

Based on in situ observations of highly intermittent SEP scattering frequency in neighboring magnetic flux tubes of solar wind, we consider an effect of such intermittency on the acceleration and transport of protons in traveling shocks. The model parameters are selected based on observations of the rising phase of major SEP events. The first modeling results presented in this Letter reveal streaming of the shock accelerated particles to far upstream of the shock via the fast transport channel penetrating the shock. The model requires neither an ad hoc escape boundary nor a replacement of the shock complex by an effective black-box source, but perhaps could justify both. Availability of fast transport channels may be one of the essential factors for the prompt occurrence of major SEP events in the interplanetary space.

This research was supported by the Academy of Finland through grant 260596 and by the UK Science and Technology Facilities Council (grant ST/J001341/1).

\section{REFERENCES}

Afanasiev, A., \& Vainio, R. 2013, ApJS, 207, 29

Axford, W. I., Leer, E., \& Skadron, G. 1977, Proc. 15th Internat. Cosmic Ray Conf. (Plovdiv) (Vol. 11; Sofia: B'Igarska Akademiia na Naukite), 132

Baring, M. G., Ellison, D. C., \& Jones, F. C. 1995, AdSpR, 15, 397

Bell, A. R. 1978, MNRAS, 182, 147

Blandford, R. D., \& Ostriker, J. P. 1978, ApJL, 221, L29

Burger, R. A., Potgieter, M. S., \& Heber, B. 2000, JGR, 105, 27447

Caprioli, D., Amato, E., \& Blasi, P. 2010, APh, 33, 307

de Hoffmann, F., \& Teller, E. 1950, PhRv, 80, 692

Dröge, W., Kartavykh, Y. Y., Klecker, B., \& Kovaltsov, G. A. 2010, ApJ, 709, 912

Drury, L. O'C. 1983, RPPh, 46, 973

Giacalone, J., \& Jokipii, J. R. 1999, ApJ, 520, 204

Heras, A. M., Sanahuja, B., Smith, Z. K., Detman, T., \& Dryer, M. 1992, ApJ, 391,359

Jones, F. C., Baring, M. G., \& Ellison, D. C. 1993, in Proc. 23rd International Cosmic Ray Conference, ed. D. A. Leahy, R. B. Hickws, \& D. Venkatesan (Vol. 2; Singapore: World Scientific), 243

Kallenrode, M.-B., \& Wibberenz, G. 1997, JGR, 102, 22311

Kocharov, L., Saloniemi, O., Torsti, J., et al. 2007a, ApJ, 659, 780

Kocharov, L., Saloniemi, O., Torsti, J., Kovaltsov, G., \& Riihonen, E. 2007b, ApJ, 654, 1121

Kocharov, L., Vainio, R., Pomoell, E., et al. 2012, ApJ, 753, 87

Krymsky, G. F. 1977, DoSSR, 234, 1306

Laitinen, T., Dalla, S., \& Marsh, M. S. 2013, ApJL, 773, L29

Lee, M. A. 2005, ApJS, 158, 38

Lee, M. A. 2006, in Geophys. Monogr. Ser., Vol. 165, ed. N. Gopalswamy, R. Mewaldt, \& J. Torsti (Washington, DC: AGU), 245

Matthaeus, W. H., Qin, G., Bieber, J. W., \& Zank, G. P. 2003, ApJL, 590, L53

Reames, D. V., \& Ng, C. K. 1998, ApJ, 505, 1002

Reville, B., Firk, J. G., \& Duffy, R. 2009, ApJ, 694, 951

Schlickeiser, R., \& Steinacker, J. 1989, SoPh, 122, 29

Toptygin, I. N. 1985, Geophys. Astrophys. Monogr., Vol. 27, Cosmic Rays in Interplanetary Magnetic Fields (Dordrecht: Reidel), 387

Torsti, J., Riihonen, E., \& Kocharov, L. 2004, ApJL, 600, L83

Vainio, R. 2003, A\&A, 406, 735

Vainio, R., \& Laitinen, T. 2007, ApJ, 685, 622

Vainio, R., \& Schlickeiser, R. 1999, A\&A, 343, 303

Zank, G. P., Rice, W. K. M., \& Wu, C. C. 2000, JGR, 105, 25079

Zhang, M., Qin, G., \& Rassoul, H. 2009, ApJ, 692, 109 